\title{
The Impact of Restaurant Coupon Removal on Diners' Consumption Behavior: A Theoretical Analysis
}

\author{
Tin-Chun Lin \\ School of Business and Economics, Indiana University, Bloomington, USA \\ Email: tinlin@iun.edu
}

How to cite this paper: Lin, T.-C. (2019) The Impact of Restaurant Coupon Removal on Diners' Consumption Behavior: A Theoretical Analysis. Theoretical Economics Letters, 9, 9-20. https://doi.org/10.4236/tel.2019.91002

Received: December 6, 2018

Accepted: January 7, 2019

Published: January 10, 2019

Copyright $\odot 2019$ by author(s) and Scientific Research Publishing Inc. This work is licensed under the Creative Commons Attribution International License (CC BY 4.0).

http://creativecommons.org/licenses/by/4.0/

\section{(c) (i) Open Access}

\begin{abstract}
We investigated how customers respond when a restaurant stops issuing coupons after making them available to customers for a period of time. Our theoretical analysis suggests that this practice will decrease customers' consumption demand and utility to a level even lower than the pre-coupon level. Customers who used the coupons and enjoyed the lower prices would suddenly feel less appreciated by a restaurant that stops issuing coupons. A demotion cost would result in a lower operating budget and a decrease in customers-to levels even lower than pre-coupon levels. Our theoretical analysis suggests that customers will be more sensitive to price increases due to the absence of coupons, rendering customer demand more elastic and the demand curve flatter.
\end{abstract}

\section{Keywords}

Coupons, Demotion Cost, Price Elasticity of Demand, Consumer Behavior

\section{Introduction}

Researchers, including $\mathrm{Qu}$ [1], Chang [2], and Lin [3], have shown that coupons can be an effective marketing strategy in attracting new customers and retaining existing customers. Coupon distribution can increase business sales and save customers money. Several interesting and important questions are raised here for investigation and discussion.

Once a restaurant stops issuing coupons to customers, how will customers respond? Will they return to their initial consumption and satisfaction levels pre-coupon? If not, will they become more sensitive to price changes? That is, will they have a higher price elasticity of demand? 
Recent research by Lin [4] relates to this topic. Lin looked at how customers responded to restaurant owners'/managers' handling of customers' unexpected incidents. Results showed that customers who were unhappy when restaurant owners/managers denied them the use of effective coupons were less likely to revisit the same restaurant. That is, customers' intention to revisit the same restaurant was frustrated by the experience of coupon denial. His experimental result implies that customers do not resume initial consumption and satisfaction levels without coupons, making them less likely to revisit the same restaurant. In the same year, Lin [5] further derived the optimal coupon discount rate by using the model of third-degree price discrimination. In his article, he pointed out an important implied issue that consumers would become more sensitive to price change after they used coupons for a while. Although Lin provided explanations, he did not offer any theoretical/empirical evidence to demonstrate the fact. For that reason, in this study, we investigated how customers would respond to coupon removal by a restaurant, and the reason for their response.

We used the theory of consumer choice to construct the model and drive optimal equilibriums. Next, we discuss the optimal equilibriums and propositions stemming from this issue.

\section{The Theoretical Framework}

\subsection{The Utility Function}

We assume that a rational consumer's objective is to maximize his/her utility when consuming goods. Suppose that the consumer always consumes two goods: restaurant meals $(X)$ and grocery foods $(Y)$ in his/her regular consumption activities. The consumer is satisfied by consuming these two goods-dining at restaurant and shopping at a grocery store. In addition, the quality of the restaurant environment $(E)$, the taste of the restaurant food $(F)$, and the quality of servers' services $(S)$ also would influence the consumer's utility when he/she dines at the restaurant. While the quality of the restaurant environment, taste of its food, and quality of servers' services will affect the consumer's utility, they cannot be determined by the consumer. That is, the consumer only can determine the number of restaurant meals and number of foods purchased at the grocery store. For this reason, these two factors ( $X$ and $Y$ ) are regarded as variables, while the other three factors $(E, F$, and $S$ ) are regarded as constant terms. Since $E, F$, and $S$ are constant, we let $\Phi=E^{\alpha} F^{\beta} S^{\gamma}$, where $\alpha, \beta$, and $\gamma$ are constant parameters and shares of the quality of restaurant environment $(E)$, taste of restaurant food $(F)$, and quality of servers' services $(S)$, respectively, and $0<\alpha, \beta, \gamma<1$.

Moreover, the consumer's utility function is assumed to display the Cobb-Douglas form, which can be shown as:

$$
U=(\Phi X)^{a} Y^{b}=\Phi^{a} X^{a} Y^{b}
$$

where $a$ and $b$ are constant parameters and shares of restaurant meals $(X)$ and grocery foods $(Y)$. In addition, $U_{X}, U_{Y}>0 ; U_{X X}, U_{Y Y}<0$; and $U_{X X}=U_{Y Y}>0$. 


\subsection{The Budget Constraint Line}

In this subsection, we show three budget constraint lines: 1) never using coupons, 2) using coupons, and 3) coupon unavailability after being available for a while.

\section{Situation 1: Never Using Coupons}

We assume that the consumer dines at a well-known chain restaurant (e.g., Olive Garden) rather than a fast food restaurant (e.g., McDonald's). Hence, servers in the chain restaurant will take orders from diners, bring dishes/drinks to diners, and clean up tables for diners. For that reason, the consumer pays not only for the price of the meal $\left(P_{x}\right)$ but also the sales tax (the sales tax rate $=\tau$ ), and tip (the tipping rate $=t$ ). Thus, the final total payment for the restaurant meal is:

$$
P_{X}=P_{x}+P_{x} \tau+P_{x}(1+\tau) t=P_{x}(1+\tau+t+\tau t) .
$$

This consumer may also shop at a grocery store. Suppose that there are no sales taxes on groceries. Therefore, the consumer pays only for the cost of the food $\left(P_{y}\right)$; hence, the final cost of grocery food is: $P_{Y}=P_{y}$.

Assume that the consumer only consumes these two goods so that his/her total expenditures on these two goods ( $X$ and $Y$ ) will be equal to his/her total budget for these two goods $(M)$. Consequently, the consumer's budget constraint line in this case can be expressed as follows:

$$
M=P_{X} \cdot X+P_{Y} \cdot Y=P_{x}(1+\tau+t+\tau t) \cdot X+P_{y} \cdot Y .
$$

\section{Situation 2: Using Coupons}

Suppose that the restaurant randomly issues coupons to customers. Luckily, the consumer receives coupons issued by the restaurant. The consumer uses the coupon (the coupon discount rate $=\phi$ ) when he/she dines at the restaurant. Therefore, with the coupon, the final total cost of the restaurant meal becomes:

$$
P_{X}=P_{x}(1-\phi)+P_{x} \tau+P_{x}(1+\tau) t=P_{x}(1-\phi+\tau+t+\tau t) .
$$

The consumer still shops at the grocery food store and pays for food $\left(P_{y}\right)$. Again, the assumption of no sales taxes on groceries is still applied to this case. The consumer's budget constraint line in this case can be displayed as below:

$$
M=P_{X} \cdot X+P_{Y} \cdot Y=P_{x}(1-\phi+\tau+t+\tau t) \cdot X+P_{y} \cdot Y .
$$

Situation 3: Coupon Unavailability after Being Available for a While

Suppose that the restaurant suddenly stops issuing coupons to customers after giving them out for a period of time. Customers who frequently used coupons and enjoyed the lower prices would suddenly feel less appreciated by this decision. The lack of appreciation could be characterized as a demotion cost $(\Omega)$ that would be generated for diners who feel demoted by the restaurant. The demotion cost is invisible but it exists, leading the consumer to reduce his/her initial budget $(M)$. In addition, the demotion cost $(\Omega)$ relates to a diner's personality \& emotionality $(\delta)$. That is, some diners may strongly feel demoted, while some diners may not strongly feel demoted or just feel okay. For that reason, the demotion cost $(\Omega)$ is a function of a diner's personality \& emotionality $(\delta)$, i.e., 
$\Omega=\Omega(\delta), \Omega^{\prime}>0$, and $0 \leq \Omega(\delta) \leq M$.

Without a coupon, the consumer's final bill for a restaurant meal will be the same as Equation (2): $P_{X}=P_{x}+P_{x} \tau+P_{x}(1+\tau) t=P_{x}(1+\tau+t+\tau t)$, but the consumer's budget constraint line will be somewhat different from the budget constraint line for never having used coupons (as shown in Equation (3): $\left.M=P_{X} \cdot X+P_{Y} \cdot Y=P_{x}(1+\tau+t+\tau t) \cdot X+P_{Y} \cdot Y\right)$. Due to the demotion cost, the consumer's budget constraint line can be specified as:

$$
M-\Omega=P_{X} \cdot X+P_{Y} \cdot Y=P_{x}(1+\tau+t+\tau t) \cdot X+P_{y} \cdot Y .
$$

We summarize these three types of budget constraint lines in Table 1.

\subsection{Equilibrium}

Choosing $X$ and $Y$ can solve the consumer's optimization problem, which maximizes the consumer's utility (shown in Equation [1]) subject to the consumer's budget constraint line (shown in Equations [3], [5] or [6]). We show these three types of equilibrium as follows.

Situation 1: Never Using Coupons

Max: $U=\Phi^{a} X^{a} Y^{b}$

S.T.: $P_{x}(1+\tau+t+\tau t) \cdot X+P_{y} Y=M$

The first-order conditions for the constrained maximum can be shown as follows:

$$
\begin{aligned}
& \frac{a \Phi^{a} X^{a-1} Y^{b}}{P_{x}(1+\tau+t+\tau t)}=\frac{b \Phi^{a} X^{a} Y^{b-1}}{P_{y}}, \text { and } \\
& M=P_{x}(1+\tau+t+\tau t) \cdot X+P_{y} \cdot Y .
\end{aligned}
$$

Based upon Equations (7) and (8), we can solve the equilibriums of these two goods:

$$
\begin{gathered}
X^{*}=\frac{M}{P_{x}(1+\tau+t+\tau t)\left(\frac{a+b}{a}\right)}=X\left(\bar{\tau}, \bar{t}, \bar{P}_{x}, \stackrel{+}{M}\right), \text { and } \\
Y^{*}=\frac{M}{P_{y}\left(\frac{a+b}{b}\right)}=Y\left(\bar{P}_{y}, \stackrel{+}{M}\right) .
\end{gathered}
$$

We then plug $X^{*}$ and $Y^{*}$ into the utility function $(U)$, which can be solved as:

$$
\begin{aligned}
U^{*} & =\left(E^{\alpha} F^{\beta} S^{\gamma}\right)^{a}\left(\frac{M}{\frac{a+b}{a}}\right)^{a+b}\left(\frac{1}{P_{x}}\right)^{a}\left(\frac{1}{P_{y}}\right)^{b}\left(\frac{1}{1+\tau+t+\tau t}\right)^{a} \\
& =U\left(\stackrel{+}{E}, \stackrel{+}{F}, \stackrel{+}{S}, \stackrel{+}{M}, \vec{P}_{x}, P_{y},-\bar{\tau}, t\right)
\end{aligned}
$$

Situation 2: Using Coupons

Max: $U=\Phi^{a} X^{a} Y^{b}$

S.T.: $P_{x}(1-\phi+\tau+t+\tau t) \cdot X+P_{y} \cdot Y=M$ 
Table 1. Three budget constraint line situations.

\begin{tabular}{lc}
\hline \multicolumn{1}{c}{ Situations } & Budget Constraint Line \\
\hline 1) Never Using Coupons & $P_{x}(1+\tau+t+\tau t) \cdot X+P_{y} Y=M$ \\
2) Using Coupons & $P_{x}(1-\phi+\tau+t+\tau t) \cdot X+P_{y} \cdot Y=M$ \\
3) Coupons Unavailable after Being Available & $P_{x}(1+\tau+t+\tau t) \cdot X+P_{y} \cdot Y=M-\Omega$ \\
\hline
\end{tabular}

The first-order conditions for the constrained maximum can be shown as follows:

$$
\begin{aligned}
& \frac{a \Phi^{a} X^{a-1} Y^{b}}{P_{x}(1-\phi+\tau+t+\tau t)}=\frac{b \Phi^{a} X^{a} Y^{b-1}}{P_{y}}, \text { and } \\
& M=P_{x}(1-\phi+\tau+t+\tau t) \cdot X+P_{y} \cdot Y
\end{aligned}
$$

Based upon Equations (12) and (13), we can solve the equilibriums of these two goods:

$$
\begin{gathered}
X^{*}=\frac{M}{P_{x}(1-\phi+\tau+t+\tau t)\left(\frac{a+b}{a}\right)}=X\left(\stackrel{+}{\phi}, \bar{\tau}, \bar{t}, \stackrel{-}{P}_{x}, \stackrel{+}{M}\right), \text { and } \\
Y^{*}=\frac{M}{P_{y}\left(\frac{a+b}{b}\right)}=Y\left(\bar{P}_{y}, \stackrel{+}{M}\right) .
\end{gathered}
$$

We then plug $X^{*}$ and $Y^{*}$ into the utility function ( $U$ ), which can be solved as:

$$
\begin{aligned}
& U^{*}=\left(E^{\alpha} F^{\beta} S^{\gamma}\right)^{a}\left(\frac{M}{\frac{a+b}{a}}\right)^{a+b}\left(\frac{1}{P_{x}}\right)^{a}\left(\frac{1}{P_{y}}\right)^{b}\left(\frac{1}{1-\phi+\tau+t+\tau t}\right)^{a} \\
& =U\left(\stackrel{+}{E}, \stackrel{+}{F}, \stackrel{+}{S}, \stackrel{+}{M}, \bar{P}_{x}, \bar{P}_{y}, \bar{\tau}, \bar{t}, \stackrel{+}{\phi}\right)
\end{aligned}
$$

Situation 3: Coupon Unavailability after Being Available for a While

Max: $U=\Phi^{a} X^{a} Y^{b}$

S.T.: $P_{x}(1+\tau+t+\tau t) \cdot X+P_{y} \cdot Y=M-\Omega$

The first-order conditions for the constrained maximum can be shown as follows:

$$
\begin{gathered}
\frac{a \Phi^{a} X^{a-1} Y^{b}}{P_{x}(1+\tau+t+\tau t)}=\frac{b \Phi^{a} X^{a} Y^{b-1}}{P_{y}}, \text { and } \\
M-\Omega=P_{x}(1+\tau+t+\tau t) \cdot X+P_{y} \cdot Y .
\end{gathered}
$$

Based upon Equations (17) and (18), we can solve the equilibriums of these two goods:

$$
X^{*}=\frac{M-\Omega}{P_{x}(1+\tau+t+\tau t)\left(\frac{a+b}{a}\right)}=X\left(\bar{\tau}, \bar{t}, \overline{P_{x}}, \stackrel{+}{M}, \bar{\Omega}\right), \text { and }
$$




$$
Y^{*}=\frac{M-\Omega}{P_{y}\left(\frac{a+b}{b}\right)}=Y\left(\stackrel{-}{P}_{y}, \stackrel{+}{M}, \stackrel{-}{\Omega}\right) .
$$

We then plug $X^{*}$ and $Y^{*}$ into the utility function $(U)$, which can be solved as:

$$
\begin{aligned}
U^{*} & =\left(E^{\alpha} F^{\beta} S^{\gamma}\right)^{a}\left(\frac{M-\Omega}{\frac{a+b}{a}}\right)^{a+b}\left(\frac{1}{P_{x}}\right)^{a}\left(\frac{1}{P_{y}}\right)^{b}\left(\frac{1}{1+\tau+t+\tau t}\right)^{a} \\
& =U\left(\stackrel{+}{E}, \stackrel{+}{F}, \stackrel{+}{S}, \stackrel{+}{M}, \vec{\Omega}, \vec{P}_{x}, \bar{P}, \bar{\tau},-\bar{t}\right)
\end{aligned}
$$

\subsection{Comparative Static Analysis}

In this subsection, we show the comparative static analysis. First, we totally differentiate Equations (12) and (13) and let $I=M-\Omega$. As a result, we obtain:

$$
\begin{aligned}
& {\left[\begin{array}{ccc}
P_{y} U_{X X}-P_{x} \delta U_{Y X} & P_{y} U_{X Y}-P_{x} \delta U_{Y Y} \\
& P_{x} \delta & P_{y}
\end{array}\right]\left[\begin{array}{l}
\mathrm{d} X \\
\mathrm{~d} Y
\end{array}\right]} \\
& =\left[\begin{array}{cccccc}
0 & \delta U_{Y} & -U_{X} & P_{x} \delta U_{Y}(1+t) & P_{x} \delta U_{Y}(1+\tau) & -P_{x} \delta U_{Y} \\
1 & -\delta X & -Y & -P_{x} X \delta(1+t) & -P_{x} X \delta(1+\tau) & P_{x} \delta X
\end{array}\right]\left[\begin{array}{c}
\mathrm{d} I \\
\mathrm{~d} P_{x} \\
\mathrm{~d} P_{y} \\
\mathrm{~d} \tau \\
\mathrm{d} t \\
\mathrm{~d} \phi
\end{array}\right]
\end{aligned}
$$

where $\delta=1-\phi+\tau+t+\tau t$,

$U_{X}=a\left(E^{\alpha} F^{\beta} S^{\gamma}\right)^{a} X^{a-1} Y^{b}$

$U_{X Y}=a b\left(E^{\alpha} F^{\beta} S^{\gamma}\right)^{\alpha} X^{a-1} Y^{b-1}=U_{Y X}$,

$U_{X X}=a(a-1)\left(E^{\alpha} F^{\beta} S^{\gamma}\right)^{a} X^{a-2} Y^{b}$,

$U_{Y}=b\left(E^{\alpha} F^{\beta} S^{\gamma}\right)^{a} X^{a} Y^{b-1}$, and

$U_{Y Y}=b(b-1)\left(E^{\alpha} F^{\beta} S^{\gamma}\right)^{\alpha} X^{a} Y^{b-2}$.

Let $|D|$ be the determinant of the pre-multiplied matrix of vector $\left[\begin{array}{ll}\mathrm{d} X & \mathrm{~d} Y\end{array}\right]$, which is:

$$
|D|=\left|\begin{array}{cc}
P_{y} U_{X X}-P_{x} \delta U_{Y X} & P_{y} U_{X Y}-P_{x} \delta U_{Y Y} \\
P_{x} \delta & P_{y}
\end{array}\right|=\left|\begin{array}{cc}
- & + \\
+ & +
\end{array}\right|<0 .
$$

Second, using Cramer's rule, the straightforward comparative static analysis yields:

$$
\begin{aligned}
& \frac{\mathrm{d} X}{\mathrm{~d} I}=\frac{\left|\begin{array}{ll}
0 & P_{y} U_{X Y}-P_{x} \delta U_{Y Y} \\
1 & P_{y}
\end{array}\right|}{|D|}>0, \\
& \frac{\mathrm{d} Y}{\mathrm{~d} I}=\frac{\left|\begin{array}{cc}
P_{y} U_{X X}-P_{x} \delta U_{Y X} & 0 \\
1 & 1
\end{array}\right|}{|D|}>0,
\end{aligned}
$$




$$
\begin{aligned}
& \frac{\mathrm{d} X}{\mathrm{~d} P_{x}}=\frac{\left|\begin{array}{cc}
\delta U_{Y} & P_{y} U_{X Y}-P_{x} \delta U_{Y Y} \\
X & -P_{y}
\end{array}\right|}{|D|}<0, \\
& \frac{\mathrm{d} Y}{\mathrm{~d} P_{x}}=\frac{\left|\begin{array}{cc}
P_{y} U_{X X}-P_{x} \delta U_{Y X} & \delta U_{Y} \\
P_{x} \delta & -\delta X
\end{array}\right|}{|D|}>\text { or }<0, \\
& \frac{\mathrm{d} X}{\mathrm{~d} P_{y}}=\frac{\left|\begin{array}{cc}
-U_{X} & P_{y} U_{X Y}-P_{x} \delta U_{Y Y} \\
-Y & -P_{y}
\end{array}\right|}{|D|}>\text { or }<0, \\
& \frac{\mathrm{d} Y}{\mathrm{~d} P_{y}}=\frac{\left|\begin{array}{cc}
P_{y} U_{X X}-P_{x} \delta U_{Y X} & -U_{X} \\
P_{x} \delta & -Y
\end{array}\right|}{|D|}<0, \\
& \frac{\mathrm{d} X}{\mathrm{~d} \tau}=\frac{\left|\begin{array}{cc}
P_{x} \delta U_{Y}(1+t) & P_{y} U_{X Y}-P_{x} \delta U_{Y Y} \\
-P_{x} X \delta(1+t) & P_{y}
\end{array}\right|}{|D|}<0, \\
& \frac{\mathrm{d} Y}{\mathrm{~d} \tau}=\frac{\left|\begin{array}{cc}
P_{y} U_{X X}-P_{x} \delta U_{Y X} & P_{x} \delta U_{Y}(1+t) \\
P_{x} \delta & -P_{x} X \delta(1+t)
\end{array}\right|}{|D|}>\text { or }<0, \\
& \frac{\mathrm{d} X}{\mathrm{~d} t}=\frac{\left|\begin{array}{cc}
P_{x} \delta U_{Y}(1+\tau) & P_{y} U_{X Y}-P_{x} \delta U_{Y Y} \\
-P_{x} X \delta(1+\tau) & P_{y}
\end{array}\right|}{|D|}<0, \\
& \frac{\mathrm{d} Y}{\mathrm{~d} t}=\frac{\left|\begin{array}{cc}
P_{y} U_{X X}-P_{x} \delta U_{Y X} & P_{x} \delta U_{Y}(1+\tau) \\
P_{x} \delta & -P_{x} X \delta(1+\tau)
\end{array}\right|}{|D|}>\text { or }<0, \\
& \frac{\mathrm{d} X}{\mathrm{~d} \phi}=\frac{\left|\begin{array}{cc}
-P_{x} \delta U_{Y} & P_{y} U_{X Y}-P_{x} \delta U_{Y Y} \\
P_{x} \delta X & P_{y}
\end{array}\right|}{|D|}>0 \text {, and } \\
& \frac{\mathrm{d} Y}{\mathrm{~d} \phi}=\frac{\left|\begin{array}{cc}
P_{y} U_{X X}-P_{x} \delta U_{Y X} & -P_{x} \delta U_{Y} \\
P_{x} \delta & P_{x} \delta X
\end{array}\right|}{|D|}>\text { or }<0 .
\end{aligned}
$$

Since $I=M-\Omega$, Equations (22) and (23) imply that $\frac{\mathrm{d} X}{\mathrm{~d} M}>0, \frac{\mathrm{d} Y}{\mathrm{~d} M}>0$, $\frac{\mathrm{d} X}{\mathrm{~d} \Omega}<0$, and $\frac{\mathrm{d} Y}{\mathrm{~d} \Omega}<0$. Intuitively, as displayed in Equations (24) and (25), a rise in the cost of a restaurant meal lessens a consumer's demand for restaurant meals but does not provide unambiguous information about grocery foods. Similarly, as shown in Equations (26) and (27), an increase in the price of groceries discourages demand for groceries and adds to uncertainty about restaurant meals. Moreover, as Equations (22) and (23) present, an increase in the con- 
sumer's maximum budget enlarges demand for both restaurant meals and grocery foods. In addition, as shown in Equations (28), (29), (30), and (31), an increase in the sales tax rate and tipping rate lessens a consumer's demand for restaurant meals but provides equivocal information about groceries. Finally, as Equations (32) and (33) show, a rise in the coupon discount rate encourages demands for restaurant meals but offers vague information about groceries.

\section{Propositions}

\section{Proposition 1:}

Diners would be encouraged to use coupons issued by the restaurant, and in doing so would increase their consumption of and demand for restaurant meals and thus raise their utility. However, once coupons become unavailable, diners would be less likely to consume restaurant meals, lowering their consumption to pre-coupon levels. Hence their utility also would drop from a higher utility to a lower utility, a utility that is even lower than the initial utility-that is, before they initially received coupons.

\section{Proof:}

As shown in Equations (9), (14), and (19), we compared the consumer's consumption demand for restaurant meals in hree different situations: never use coupons, use coupons, and coupon unavailable after being available. As a result,

\section{Use Coupon:}

$$
\frac{M}{P_{x}(1-\phi+\tau+t+\tau t)\left(\frac{a+b}{a}\right)}>
$$

\section{Never Use Coupon:}

$$
\frac{M}{P_{x}(1+\tau+t+\tau t)\left(\frac{a+b}{a}\right)}>
$$

Coupon Unavailable after being Available:

$$
\frac{M-\Omega}{P_{x}(1+\tau+t+\tau t)\left(\frac{a+b}{a}\right)}
$$

Moreover, as shown in Equations (11), (16), and (21), we compared the consumer's utility in these three different situations: never use coupons, use coupons, and coupons unavailable after being available. Consequently,

Use Coupon:

$$
\left(E^{\alpha} F^{\beta} S^{\gamma}\right)^{a}\left(\frac{M}{\frac{a+b}{a}}\right)^{a+b}\left(\frac{1}{P_{x}}\right)^{a}\left(\frac{1}{P_{y}}\right)^{b}\left(\frac{1}{1-\phi+\tau+t+\tau t}\right)^{a}>
$$

\section{Never Use Coupon:}




$$
\left(E^{\alpha} F^{\beta} S^{\gamma}\right)^{a}\left(\frac{M}{\frac{a+b}{a}}\right)^{a+b}\left(\frac{1}{P_{x}}\right)^{a}\left(\frac{1}{P_{y}}\right)^{b}\left(\frac{1}{1+\tau+t+\tau t}\right)^{a}>
$$

\section{Coupon Unavailable after being Available:}

$$
\left(E^{\alpha} F^{\beta} S^{\gamma}\right)^{a}\left(\frac{M-\Omega}{\frac{a+b}{a}}\right)^{a+b}\left(\frac{1}{P_{x}}\right)^{a}\left(\frac{1}{P_{y}}\right)^{b}\left(\frac{1}{1+\tau+t+\tau t}\right)^{a} .
$$

Graphically, as shown in Figure 1(a), before the restaurant issues coupons to customers, the diner's initial budget constraint line is $i k$. Thus, the diner's initial consumption demand for restaurant meals is $X 1$ and his/her level of satisfaction (utility) is at U1. After the diner uses the restaurant-issued coupons, the diner's new budget constraint line shifts to $i j$. Hence, the diner increases his/her consumption demand for restaurant meals to $X 2(X 2>X 1)$ and raises his/her level of satisfaction (utility) to U2 (U2 > U1). However, when the restaurant suddenly stops issuing coupons to customers after diners have used them for a while, the diner's budget constraint line moves to $m n$. Therefore, the diner decreases his/her consumption demand for restaurant meals to $X 3(X 3<X 1<X 2)$ and drops his/her level of satisfaction (utility) to U3 (U3 < U1 < U2). Q.E.D.

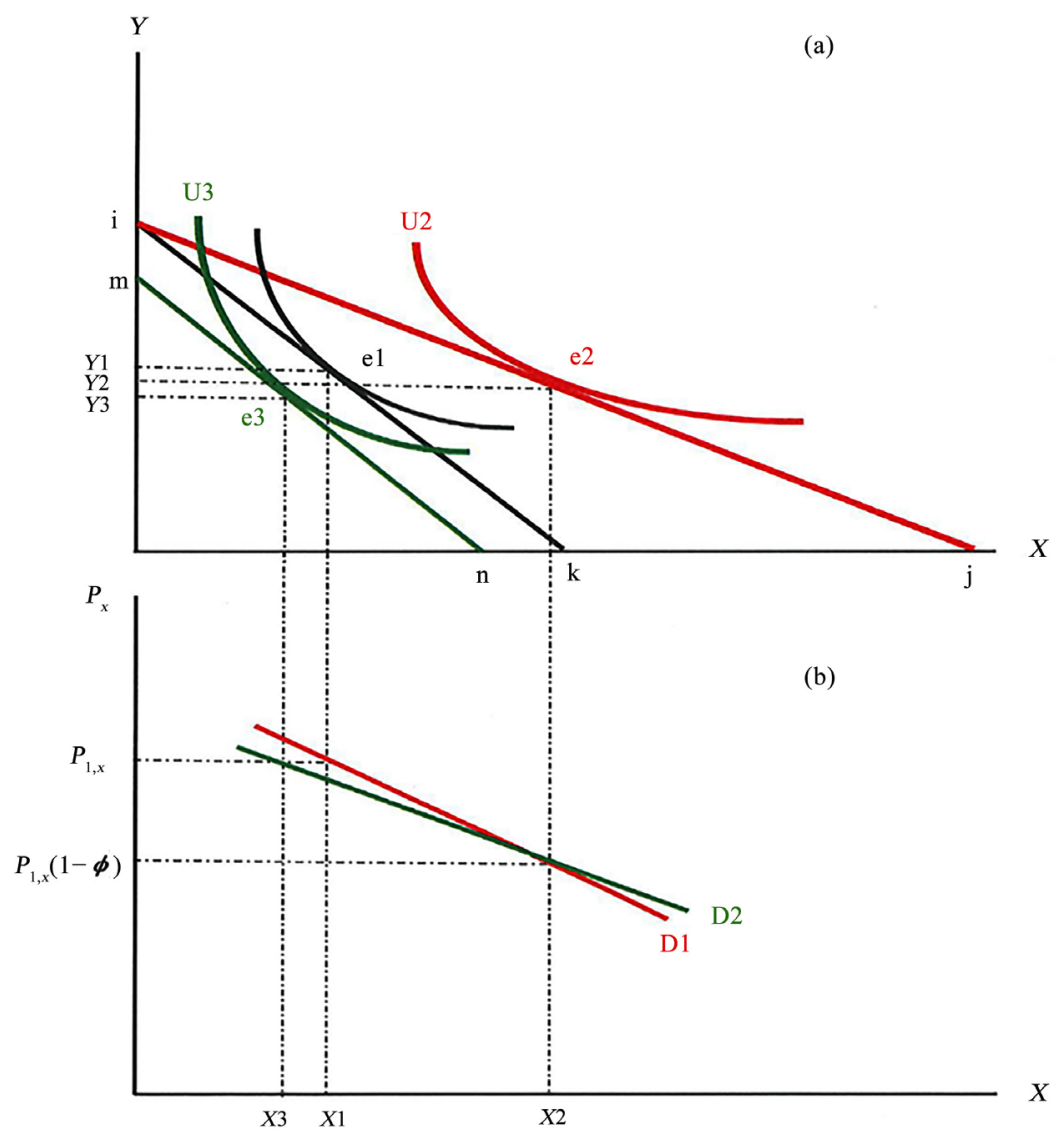

Figure 1. (a) indifference curves and equilibriums; (b) demand curves. 
Proposition 1 points out an important fact: after consumers receive and use the coupon promotion from the restaurant, their satisfaction level may not return to the initial pre-coupon satisfaction level once the coupons are no longer available. This is because customers would feel unappreciated by the restaurant and hence a demotion cost could be created for customers. This cost could cause customers to decrease their consumption demand to a level even lower than the pre-coupon level. Their satisfaction level also would drop to a level even lower than that manifested pre-coupon.

In addition, in November 2016 we informally interviewed the owner of a buffet restaurant in Indiana (note: the restaurant owner did not want us to disclose his restaurant's name in our paper, thus we are not able to share that information). The owner told us that the restaurant began randomly issuing $15 \%$ discount coupons to customers in local areas in January 2014. As a result, the restaurant's total sales approximately improved $20 \%-25 \%$ on average in the following year compared to the past year's sales pre-coupon. The owner then stopped issuing coupons to customers because the owner thought restaurant sales would continue growing without coupons. Unfortunately, total sales roughly dropped $30 \%$ - 35\% on average in six months post-coupon compared with the coupon year. The percentage decrease was even higher than the percentage increase. The owner also said that during the coupon year, around $50 \%$ of customers used coupons and most were continuing customers. However, after the restaurant stopped issuing coupons to customers, those customers who used coupons returned to the restaurant much less frequently than they had in both the coupon and pre-coupon years. The information from the informal interview implied that a demotion cost might be due to coupon unavailability. This evidence is consistent with our theoretical analysis in Proposition 1.

\section{Proposition 2:}

After consumers use coupons for a while, they become more sensitive to price changes if prices increase to pre-coupon levels. Therefore, they will have a higher price elasticity of demand and their demand curve will become flatter.

\section{Proof:}

Figure 1(b) shows this proposition. Graphically, as shown in Figure 1(b), after the customer uses coupons, the price drops from $P_{1, x}$ to $P_{1, x}(1-\phi)$, but the consumption demand increases from $X 1$ to $X 2(X 2>X 1)$. However, when coupons are removed by the restaurant and the price returns to its original level $P_{1, x}$, the customer decreases his/her consumption demand from $X 2$ to $X 3$, a level even lower than the initial level $X 1(X 3<X 1<X 2)$. Therefore, the new demand curve $(D 2)$ is flatter than the initial demand curve $(D 1)$. A flatter demand curve implies a higher price elasticity of demand. Q.E.D.

Proposition 2 implies that customers who use coupons will become more sensitive to price changes after they use coupons for a while. For that reason, the price elasticity of demand for customers who used coupons will become higher than the price elasticity of demand for customers who did not use coupons. The 
reason is the same as that offered for Proposition 1-the demotion cost, as described above.

\section{Conclusions}

In this paper, we investigated consumer response to the use and subsequent unavailability of coupons at a restaurant that started and then stopped the coupon process. Our theoretical analysis suggests that customers will lower their consumption demand and utility to a pre-coupon level even lower than the initial level of consumption demand and utility. This is because customers who have used coupons and enjoyed a lower price for a while may suddenly feel unappreciated when coupons are no longer distributed by or welcome at a restaurant. These feelings may generate a demotion cost, resulting in a lower budget for restaurant excursions. Customer patronage may reach a level even lower than the initial level of pre-coupon consumption demand and utility.

Moreover, our theoretical analysis suggests that customers become more sensitive to price changes when the price is increased to the pre-coupon discount level. Their demand then becomes more elastic and the demand curve becomes flattering.

Finally, this paper is a theoretical study and offers an innovative explanation (the demotion cost) to explain why customers may lower their consumption demand and utility to a level even lower than the pre-coupon level. Our next steps for this work include conducting a survey and constructing an empirical study to verify whether or not our theoretical analysis is sustainable.

\section{Acknowledgements}

The author would like to thank Ms. Joy Deng for her editorial assistance and the anonymous referee for his/her feedback and suggestions.

\section{Conflicts of Interest}

The authors declare no conflicts of interest regarding the publication of this paper.

\section{References}

[1] Qu, H. (1997) Determinant Factors and Choice Intention for Chinese Restaurant Dining: A Multivariate Approach. Journal of Restaurant and Foodservice Marketing, 2, 35-49. https://doi.org/10.1300/J061v02n02_03 http://download.xuebalib.com/xuebalib.com.22812.pdf

[2] Chang, J. (2003) Analyzing the Impact of Sales Promotions on the Sources of Category Expansion. ProQuest Dissertations Publishing, Northwestern University, Evanston, $1-446$.

[3] Lin, T.-C. (2017) Modeling Consumer Intention to Revisit the Same Restaurant and a Simple Algorithm of Coupon Discount Rate. Theoretical Economics Letters, 7, 1179-1188. http://file.scirp.org/pdf/TEL_2017072014435686.pdf

[4] Lin, T.-C. (2018) Impact of Restaurant Owners'/Managers' Handling of Customers' 
T.-C. Lin

Unexpected Incidents on Customers' Revisit Intention. International Journal of Economics and Business Research, 15, 108-124.

https://doi.org/10.4236/tel.2017.75079

https://www.inderscience.com/info/inarticle.php?artid=88507

[5] Lin, T.-C. (2018) Deriving an Optimal Restaurant Coupon Discount Rate: Evidence and Discussion. Theoretical Economics Letters, 8, 1674-1683.

https://doi.org/10.4236/tel.2018.810108 